\title{
INTERFACE TECHNOLOGY FOR GEOMETRICALLY NONLINEAR ANALYSIS OF MULTIPLE CONNECTED SUBDOMAINS
}

\author{
Jonathan B. Ransom* \\ NASA Langley Research Center \\ Hampton, VA 23681-0001
}

\begin{abstract}
$\underline{\text { Abstract }}$
Interface technology for geometrically nonlinear analysis is presented and demonstrated. This technology is based on an interface element which makes use of a hybrid variational formulation to provide for compatibility between independently modeled connected subdomains. The interface element developed herein extends previous work to include geometric nonlinearity and to use standard linear and nonlinear solution procedures. Several benchmark nonlinear applications of the interface technology are presented and aspects of the implementation are discussed.
\end{abstract}

\section{Introduction}

Rapid detail modeling and reliable analysis methodology are needed in order to provide tractable computational capabilities early in the design of aerospace and ground vehicle structures. Although critical structural details are often not well-defined in the early preliminary design phases, achieving an understanding of the effect of certain details can greatly impact early design decisions. In addition, rapid modeling and analysis capabilities that include such design detail provide early insight into and predictions of the failure mechanisms of structural concepts. Design verification, often satisfied through refined analysis, may be impacted significantly by computational methods, simulations and techniques which predict accurately detailed stress states such as yield and fracture stresses. Detail stress analysis often relies heavily on the finite element method which requires fine mesh discretizations of the structure in the vicinity of stress or strain concentrations. The resulting finite element models generally contain both fine and coarse mesh discretizations. Tedious, costly and time consuming transition modeling is generally used between the subdomains of fine and coarse refinement. Such transition modeling is also typically used for assembling independent substructure models, inserting part or component detail,

- Aerospace Engineer, Member AlAA

Copyright $(1997$ by the American Institute of Aeronautics and Astronautics, Inc. No copyright is asserted in the United States under Title 17, U.S. Code. The U.S. Government has a royaltyfree license to exercise all rights under the copyright claimed herein for government purposes. All rights are reserved by copyright owner. global/local modeling and modifying structural features. The extensive labor required for such transition modeling often precludes the use of the finite element method early in design.

Recently, a method for connecting finite element models without the use of transition modeling has been developed. ${ }^{1,2}$ This method, called interface technology, is an improved technique for connecting multiple dissimilar meshed subdomains or substructures to form a single finite element model. Compared with conventional transition modeling techniques, this method allows designers to create models for structural analysis faster and with less effort while retaining computational accuracy. The method promises to significantly reduce engineer modeling time and effort, thus enabling comprehensive early design.

Unlike many global/local ${ }^{3,4}$ and substructuring ${ }^{5.7}$ approaches, the interface technology provides a means of connecting independently modeled substructures or subdomains whose nodes along a common boundary need not coincide. The technology is based on a variational formulation which enforces the compatibility between the connected subdomains in a weak sense. This compatibility provides for the interaction between the independently modeled subdomains. Conventional global/local techniques, in which global coarse models are used to provide loads or displacements ${ }^{8}$ for local refined models, do not account for full subdomain interaction including changes in the load path prediction due to the refined local modeling. In addition to global/local modeling, interface technology enables assembly of independently created model components, repair patch modeling, contact/friction/sliding applications and detail model insertion.

The interface technology has been shown to maintain solution accuracy for a wide range of applications. ${ }^{1,29,10}$ The method was developed and validated on benchmark problems in reference 1, reformulated in the form of an element in reference 2, and demonstrated on large scale structural applications in references 9 and 10 . The interface technology has been incorporated into the commercially available finite element computer code, MSC/NASTRAN. ${ }^{11,12}$ However, until recently, the interface technology has been limited to linear stress analysis. Although linear analysis is quite useful, it 
can miss important phenomena. Ignoring nonlinear effects can lead to nonconservative predictions for softening systems and severe weight penalties for stiffening systems.

Thus, the purpose of this paper is to present the extended capabilities of the interface technology to geometrically nonlinear analysis. This nonlinear capability is developed, described and demonstrated on representative structures. A new interface element solution strategy for both linear and nonlinear analysis is developed and described. The effectiveness of the approach is demonstrated using five elastic plate and shell applications including: a pressureloaded clamped square plate, a pressure-loaded clamped cylindrical panel, a point-loaded hinged cylindrical panel, a moment-loaded cantilevered plate and a compression-loaded composite panel. These application problems exhibit nonlinear responses that verify the capabilities of the technology; namely, (1) softening, (2) stiffening, (3) snapthrough, (4) snap-back, (5) large rotation behavior and (6) postbuckling. The guidelines for developing this technology include the requirements that it be compatible with general-purpose finite element codes, valid for a wide range of finite elements, costeffective and accurate for predicting the linear and nonlinear responses as well as the overall and detailed stress states of structural components.

\section{Overview of the Interface Element}

The interface element was developed in detail in reference 2 and is briefly described herein. It allows the independent modeling of different substructures or components without concern for one-to-one nodal coincidence between the finite element models. Moreover, it acts as "glue" between independent finite element models with different mesh densities and nodal layouts. Thus, the interface element provides a transition modeling role through an analytical variational procedure and avoids the use of transition meshes. It alone does not improve the quality of the finite element results obtained by a particular model but rather improves the efficiency of the modeling and makes effective use of existing finite elements. By eliminating or reducing transition modeling, the introduction of distorted elements, which may degrade the quality of the solution, is limited to what is required to represent the geometry of the structure. In addition, the elimination of unnecessary element distortion errors allows the use of a less refined mesh and therefore, the same qualitative results may be obtained with fewer degrees of freedom.

Consider, for example, the domain shown in Figure 1 and modeled as three independently discretized substructures. The interface element is dis- cretized with a mesh of evenly-spaced pseudo-nodes (open circles in the figure) which need not be coincident with any of the interface nodes (filled circles in the figure) of any of the substructures. The hybrid variational formulation ${ }^{1.2}$ employs an integral form for the compatibility between the interface line element and the finite element substructures. The displacement vector, $v$, of the interface element is assumed to be independent of the displacement vectors, $u$, of the substructures to which it is attached.

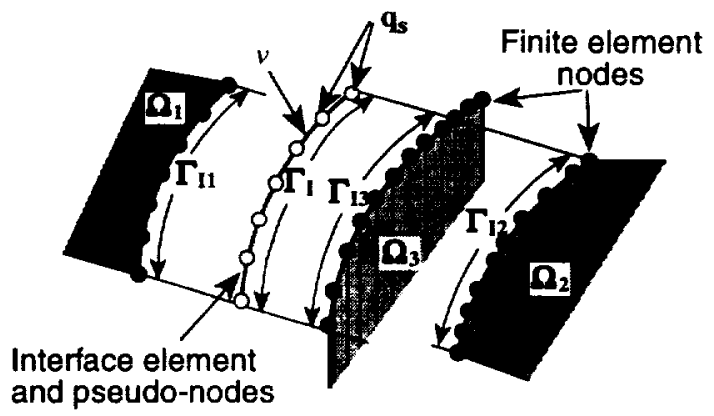

Figure 1. Typical Interface Element Definition.

\section{Nonlinear Formulation}

In the previous work presented in reference 2 , the interface element was limited to linear analyses. In the present work, the range of applicability is extended to the geometrically nonlinear regime. The approximation in the $k^{\text {ht }}$ domain, $\Omega_{k}$, is made in terms of the displacements, $u_{i j}$ and $v_{i}$, and the interface tractions, $\lambda_{i j}$. Tractions and displacements are prescribed on boundaries $S_{k}^{(\sigma)}$ and $S_{k}^{(u)}$, respectively (see Figure 2).

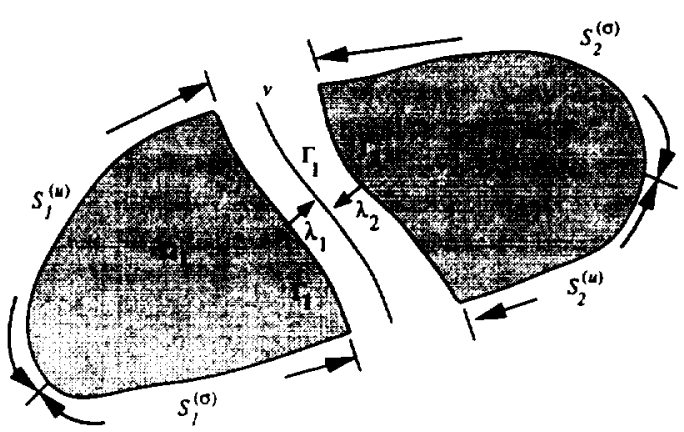

Figure 2. Linking of Two Domains Using Interface Technology. 
Using the weak form of the principle of virtual work, leads to

$$
\begin{aligned}
\delta W_{k} & =\int_{\Omega_{k}} \sigma^{T} \delta \varepsilon \mathrm{d} \Omega_{k}-\int \delta u_{k}{ }^{T} F_{k} \mathrm{~d} \Omega_{k} \\
& -\int_{\Omega_{k}} \delta u_{k} T_{\phi_{k}} \mathrm{~d} S_{k}^{(\sigma)}-\int_{\Gamma_{k}} \delta u_{k} T \lambda_{k} \mathrm{~d} \Gamma_{I} \\
& +\int_{\Gamma_{I}} \delta v_{k}{ }^{T} \lambda_{k} \mathrm{~d} \Gamma_{I}=0
\end{aligned}
$$

where for the $k^{\text {th }}$ substructure, $F_{k}$ are the external applied forces and $\phi_{k}$ are the applied tractions. As usual, the satisfaction of prescribed displacement on $s_{k}^{(u)}$ is implied by the approximation for $\boldsymbol{u}_{\boldsymbol{i}}$. The $N_{s s}$ subdomain equations are completed by enforcing compatibility through the use of the constraint integral for the $N_{l}$ interface elements

$$
\delta W_{c_{i}}=\sum_{j=1}^{n_{s s}(i)} \int_{\Gamma_{i j}} \delta \lambda_{i j}^{T}\left(v_{i}-u_{i j}\right) \mathrm{d} \Gamma_{i j}=0
$$

where $n_{s s}(i)$ is the number of substructures connected to interface element $i$. Use of this constraint integral corresponds to the "frame" method of reference 13. Therefore, for the entire domain

$$
\delta \bar{W}=\sum_{k=1}^{N_{s s}} \delta W_{k}+\sum_{i=1}^{N_{l}} \delta W_{c_{i}}=0
$$

Discretization of displacements in each domain and displacements and Lagrange multipliers on the interface yields the final system of equations. At the finite element level, the first integral term in Equation (1) may be written as

$$
\begin{aligned}
\int_{\Omega_{k}} \sigma^{T} \delta \varepsilon \mathrm{d} \Omega_{k} & =\int_{\Omega_{k}} \delta \varepsilon^{T} \sigma \mathrm{d} \Omega_{k} \\
& =\sum_{n=1}^{n e l(k)} \int_{\Omega e} \delta \varepsilon^{T} \sigma \mathrm{d} \Omega_{e}
\end{aligned}
$$

where $n e l(k)$ is the number of elements in the $k^{\text {th }}$ substructure. Equation (4) is evaluated using the nonlinear strain displacement relations and the constitutive relation for elastic, small strain behavior and may be rewritten as

$$
\begin{aligned}
\int_{\Omega e} \delta \varepsilon^{T} \sigma \mathrm{d} \Omega_{e} & =\delta q_{e}^{T}\left(k_{0}+k_{\sigma}+k_{L}\right) q_{e} \\
& =\delta q_{e}^{T}\left(k_{T}\right) q_{e}
\end{aligned}
$$

where $q_{e}$ is a vector of nodal generalized displacements for the element, $\boldsymbol{k}_{0}$ is the linear stiffness matrix, $k_{\mathfrak{o}}$ is the geometric stiffness matrix, $\boldsymbol{k}_{L}$ is the large displacement matrix, and $k_{T}$ is the tangent stiffness matrix.

Upon assembling the element contributions in each subdomain, Equation (4) may be written as

$$
\int_{\Omega_{k}} \sigma^{T} \delta \varepsilon \mathrm{d} \Omega_{k}=\delta q_{k}^{T}\left(K_{T}\right)_{k} q_{k}
$$

where $\left(K_{T}\right)_{k}$ is the tangent stiffness matrix for the $k^{\text {th }}$ substructure.

The independent approximations for the finite element displacements, interface displacements, and interface tractions are, respectively

$$
\begin{aligned}
& u_{i j}=N_{i j} \bar{q}_{i j} \\
& v_{i}=T_{i} q_{s_{i}} \\
& \lambda_{i j}=R_{i j} \alpha_{i j}
\end{aligned}
$$

where $\overline{\boldsymbol{q}}_{i j}$ and $\boldsymbol{q}_{s_{i}}$ are the nodal degrees of freedom corresponding to $\boldsymbol{u}_{i j}$ and $\boldsymbol{v}_{i}$, and $\alpha_{i j}$ are the unknown coefficients of the Lagrange multipliers, $\lambda_{y j}$. The matrix $N_{i j}$ is the matrix of finite element shape functions on substructure $j$ along interface $i, T_{t}$ is formed as a result of passing a cubic spline through the evenly-spaced pseudo-nodes, $\boldsymbol{R}_{\boldsymbol{u}}$ is formed as a result of using constant functions for linear finite elements and linear functions for quadratic finite elements.

Hence, Equation (3) may be rewritten as

$$
\begin{aligned}
\delta \bar{W} & =\sum_{k=1}^{N_{s s}} \delta q_{k}^{T}\left(K_{T}\right)_{k} q_{k}-\delta q_{k}^{T} f_{k} \\
& -\sum_{i=1}^{N_{I}} \sum_{j=1}^{n_{s s}(i)} \int_{\Gamma_{i j}} \delta q_{i j}^{T} N_{i j}^{T} R_{i j} \alpha_{i j} \mathrm{~d} \Gamma_{i j} \\
& +\sum_{i=1}^{N_{I}} \sum_{j=1}^{n_{s s}(i)} \int_{\Gamma_{i j}} \delta q_{s_{i}}^{T} T_{i}^{T} R_{i j} \alpha_{i j} \mathrm{~d} \Gamma_{i j} \\
& +\sum_{i=1}^{N_{I}} \sum_{j=1}^{n_{s s}(i)} \int_{\Gamma_{i j}} \delta \alpha_{i j}^{T} R_{i j}^{T} T_{i} q_{s_{i}} \mathrm{~d} \Gamma_{i j} \\
& -\sum_{i=1}^{N_{I}} \sum_{j=1}^{n_{s s}(i)} \int_{\Gamma_{i j}} \delta \alpha_{i j}^{T} R_{i j}^{T} N_{i j} \bar{q}_{i j} \mathrm{~d} \Gamma_{i j}=0
\end{aligned}
$$

where

$$
f_{k}=\int_{\Omega_{k}} N_{k}^{T} F_{k} \mathrm{~d} \Omega_{k}+\int_{S_{k}^{(\sigma)}} N_{k}^{T} \phi_{k} \mathrm{~d} S_{k}^{(\sigma)}
$$


and defining the interface matrices as

$$
\begin{aligned}
& M_{i j}=-\int_{\Gamma_{i j}} N_{i j}^{T} R_{i j} \mathrm{~d} \Gamma_{i j} \text { and } G_{i j}=\int_{\Gamma_{i j}} T_{i}^{T} R_{i j} \mathrm{~d} \Gamma_{i j} \\
& \text { Thus, for arbitrary } q_{k} \text { on } \Omega_{k} \\
& \sum_{k=1}^{N_{s s}}\left(K_{T}\right)_{k} q_{k}-f_{k}+\sum_{i=1}^{N_{l}} \sum_{j=1}^{n_{s s}(i)} M_{i j} \alpha_{i j}=0
\end{aligned}
$$

for arbitrary $q_{s_{i}}$ on $\Gamma_{l}$

$$
\sum_{i=1}^{N_{l}} \sum_{j=1}^{n_{s s}(i)} G_{i j} \alpha_{i j}=0
$$

for arbitrary $\alpha_{i j}$ on $\Gamma_{l}$

$$
\sum_{i=1}^{N_{I}} \sum_{j=1}^{n_{s s}(i)} G_{i j}^{T} q_{s_{i}}+M_{i j}^{T} \bar{q}_{i j}=0
$$

Thus, the resulting system of equations is given in matrix form as

$$
\left[\begin{array}{ccc}
K_{T} & 0 & M_{I} \\
0 & 0 & G_{I} \\
M_{I}^{T} & G_{I}^{T} & 0
\end{array}\right]\left\{\begin{array}{c}
q \\
q_{s} \\
\alpha
\end{array}\right\}=\left\{\begin{array}{l}
f \\
0 \\
0
\end{array}\right\}
$$

where $K_{T}, q$ and $f$ are the assembled tangent stiffness matrix, displacement vector and force vector for the entire structure, and $\boldsymbol{M}_{l}, \boldsymbol{G}_{l}, \boldsymbol{q}_{s}$ and $\alpha$ are the assembled $\boldsymbol{M}_{i}, \boldsymbol{G}_{i}, \boldsymbol{q}_{i}$, and $\alpha_{i j}$ for all interface elements. The assembled tangent stiffness matrix $K_{T}$ is a block diagonal matrix containing the tangent stiffness matrices $\left(K_{T}\right)_{k}$ of each of the substructures along its block diagonal. The interface element "stiffness" matrix and vector of unknowns are given in the same form as that given for the linear interface element of reference 2. However, in the moderate-to-large strain regime, the integral limits for the coupling terms of Equation (10) are related to the reference state (the deformed configuration in this implementation) and account for straining of the interface. This straining has been neglected herein but does not adversely affect the interface element performance for this study. The system of equations in Equation (14) is symmetric but not banded nor positive definite. Therefore, standard Cholesky solvers may not be used, unless row or column pivoting is performed to obtain the solution. The number of additional degrees of freedom associated with the interface element is generally small in comparison with the total number of degrees of freedom in the structure. Thus, modeling flexibility is provided at a relatively small computational expense. The computational expense may be reduced additionally as the efficiency of new solution algorithms for the system of equations in Equation (14) is increased. This nonlinear interface element was implemented within a general-purpose, finite element code COMET/AR. ${ }^{14}$ This implementation is briefly described in the next subsection.

\section{Nonlinear Element Implementation}

The nonlinear solution procedure employed herein is based on a Newton/Raphson incremental strategy for automatic load step control. The socalled modified Newton/Raphson method, which forms and factors the tangent stiffness matrix periodically rather than at every nonlinear iteration, has been used in the nonlinear analyses of the benchmark applications presented in the next section. A corotational formulation ${ }^{15.16}$ which identifies the reference state as the current deformed configuration is used to describe the motion. This formulation separates the rigid body motion from the strain-producing motion thus allowing for either linear or nonlinear strain-displacement relations at the finite element level. Deformations are computed based on the original configuration within the local corotated frame. An arc-length control strategy, ${ }^{17}$ the most general approach for obtaining the load-deflection response, is used to handle limit points with both snap-though and snap-back behaviors.

The standard nonlinear solution strategy has been adapted to incorporate the interface element. Interface element "stiffness" matrices are computed and assembled along with the finite element tangent stiffness matrices. The displacement solutions are used to compute the increment in the arc-length and predict the solution at the next load step. In analyses with the interface element, these displacement solutions contain coefficients of the Lagrange multipliers as well as the nodal displacements (see Equation (14)). These Lagrange multiplier coefficients are eliminated when computing the arc-length increment. In addition, in the standard procedure, the number of negative roots is used to determine the structural instability during loading as well as to identify changes in the loading direction. This is not a viable approach in this work since the indefinite system of Equation (14) could have both positive and negative roots even along the stable equilibrium path. Thus, an approach based on the change in incremental work is used herein for the interface technology rather than the number of negative roots to determine instability and change in load direction. The change in incremental work is essentially the so-called current stiffness parameter. ${ }^{18}$

\section{Numerical Results}

The capabilities of the interface element for geometrically nonlinear analysis are demonstrated on several benchmark applications. The application structures exhibit a wide variety of response charac- 
teristics, namely, stiffening, softening, snap-through, snap-back, large rotation, and postbuckling responses. These response characteristics test extensively the robustness of the nonlinear interface element implementation. A nine-node Assumed Natural-coordinate Strain (ANS) shell element ${ }^{19}$ has been used in each of the applications discussed herein. The ANS element has five degrees of freedom at each node (three displacements, two rotations, and no drilling degree of freedom). Results from each interface element analysis (denoted coupled analysis herein) are compared with a reference solution obtained by using a reference model (with no interface) in which the discretization of the most refined independent substructure model extends over the entire domain. The reference solutions are obtained from analyses performed as part of this study. These solutions, in excellent agreement with those published in the open literature, are used to assess the performance of the interface technology for the given finite element type, mesh discretization, and nonlinear solution strategy.

\section{Clamped Square Plate}

An isotropic square plate which is clamped on all four edges (shown in Figure 3 ) and is subjected to a uniform pressure load is used to demonstrate the capability of the interface technology for a structure exhibiting a stiffening response. The plate material properties are $1000 \mathrm{ksi}$ for the Young's modulus and 0.3 for the Poisson's ratio. The plate edge length, $L$, is 10 in., and the thickness, $t$, is 0.1 in. The coupled finite element model is shown in Figure 4. The interface element is located at the panel midlength (shown by the gray shaded line in Figure 4). In Figure 5, the normalized pressure load is given as a function of the transverse deflection at the center of the plate (point $\mathrm{C}$ in Figure 3 ) normalized by the plate thickness. The response curve indicates the stiffening behavior of the plate as the load is increased. The coupled analysis, utilizing an interface element as the coupling agent, is in excellent agreement with the reference solution.

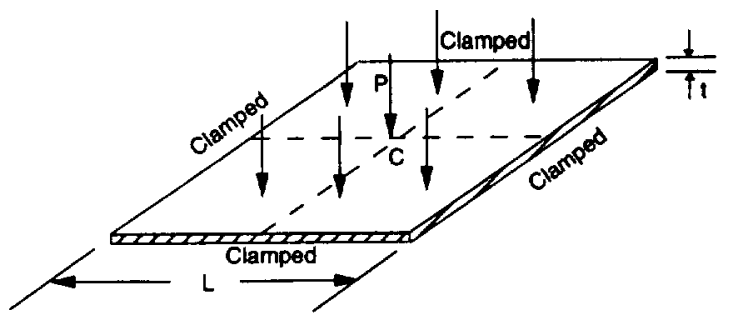

Figure 3. Clamped Square Plate Subjected to Uniform Pressure.

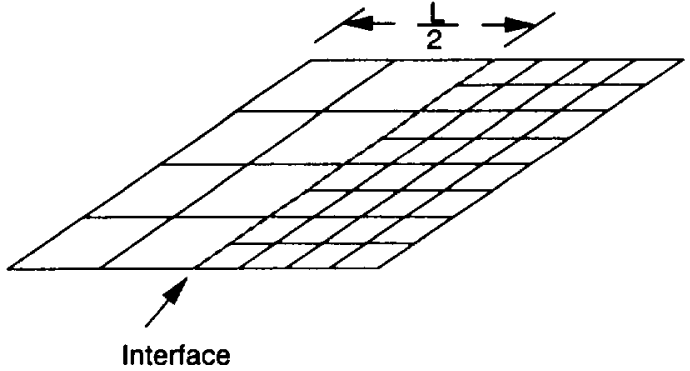

Figure 4. Finite Element Model for Clamped Square Plate.

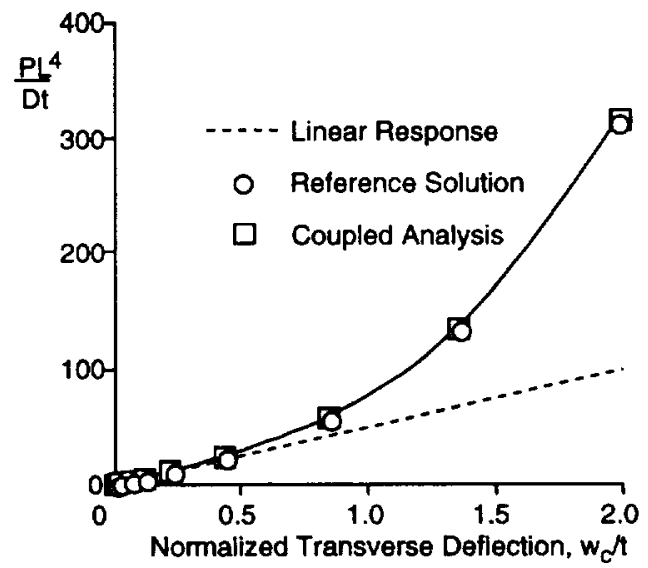

Figure 5. Central Deflection of Clamped Square Plate.

\section{Clamped Cylindrical Panel}

An isotropic cylindrical panel which is clamped on all four edges (shown in Figure 6) and is subjected to a uniform pressure load is used to demonstrate the capability of the interface technology on a shell structure as well as for a structure exhibiting a softening response. This panel configuration is given in the literature and attributed to Brebbia and Conner. ${ }^{20}$ The panel material properties are $450 \mathrm{ksi}$ for the Young's modulus and 0.3 for the Poisson's ratio. The panel planform is square with a length, L, of 20 in. and a radius, $R$, of 100 in. The panel thickness, $t$, is 0.125 in.

The finite element model shown in Figure 7 involves two independently modeled, coupled substructures. The interface element is located along an axial line at half the panel planform dimension. In Figure 8, the pressure load, $p$, is shown as a function of the transverse deflection at the center of the panel (point $\mathrm{C}$ in Figure 6). The response curve indicates the softening behavior of the panel as the load is 
increased. The coupled analysis utilizing an interface element is in excellent agreement with the reference solution.

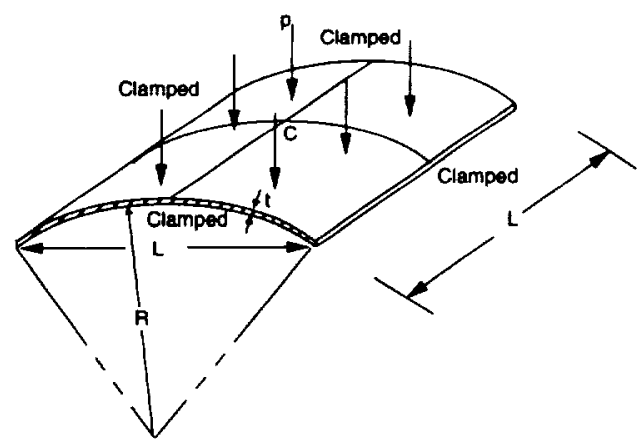

Figure 6. Clamped Cylinder Subjected to Uniform Pressure.

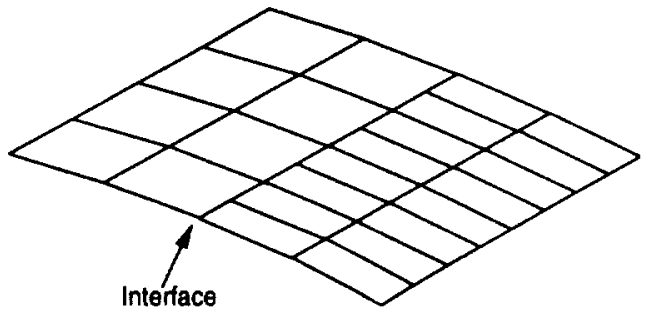

Figure 7. Finite Element Model for Clamped Cylindrical Panel.

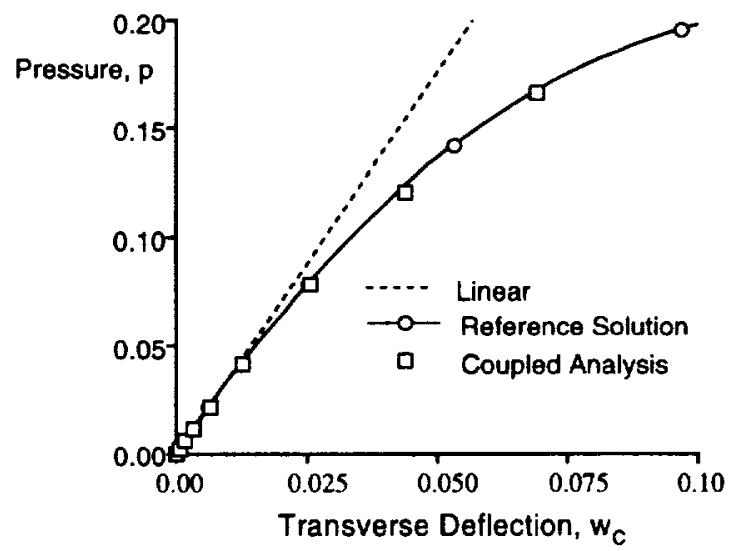

Figure 8. Central Deflection of Clamped Cylindrical Panel.

As discussed in a previous section, the corotational formulation allows for the use of either linear or nonlinear strain-displacement relations at the finite element level. (The corotational formulation includes a substantial part of the nonlinear response character by separating out the rigid body motion.) The interface technology provides the flexibility to use different strain approximations in each of the independent substructures. The effect of the nonlinear terms in the strain-displacement relations on the transverse deflection is shown in Figure 9. Results are obtained with linear and nonlinear straindisplacement relations and are denoted in Figure 9 by (L) and (NL), respectively. Three reference solutions are obtained and are given by the solid lines in the figure. The first reference solution, denoted reference (NL) in the figure, is obtained using nonlinear strain-displacement relations and the discretization of the most-refined substructure of the coupled model (see Figure 7) over the entire domain of the panel. This finite element model will be referred to hereafter as the refined reference model. The second reference solution, denoted reference ( $L$ ) in the figure, is obtained using linear strain-displacement relations and the refined reference model. The third reference solution, denoted coarse reference $(L)$ in the figure, is obtained using linear strain-displacement relations and the discretization of the less-refined substructure of the coupled model (see Figure 7) over the entire domain of the panel. This third reference solution is used to bound the results of the coupled analyses, and the finite element model used will be referred to hereafter as the coarse reference model. When linear strain approximations are used, the predicted structural behavior is not as soft as that behavior exhibited with nonlinear strain approximations. This behavior is illustrated by the comparison of the two reference solutions obtained with the refined reference model (solid circles and squares in Figure 9). This effect is magnified as the size of the elements is increased, which is evident by the results for the coarse reference model (solid triangles in the figure). The impact of the use of linear strains is decreased as the mesh is refined. An enlarged view of the results is shown in Figure $9 \mathrm{~b}$ to delineate better the effect of the strain approximation on the transverse deflection.

As was shown in Figure 8, the interface element analysis with nonlinear strain approximations (open circles in Figure 9) is in excellent agreement with the reference solution with nonlinear strain approximations. The response obtained with linear strains (open triangles in Figure 9) illustrates the stiffening of the panel due to the combined effect of the strain approximation and the coarse discretization of one of the substructures. These results are bounded by the coarse and refined reference models using linear strain approximations (solid triangles and solid squares in the figure). 


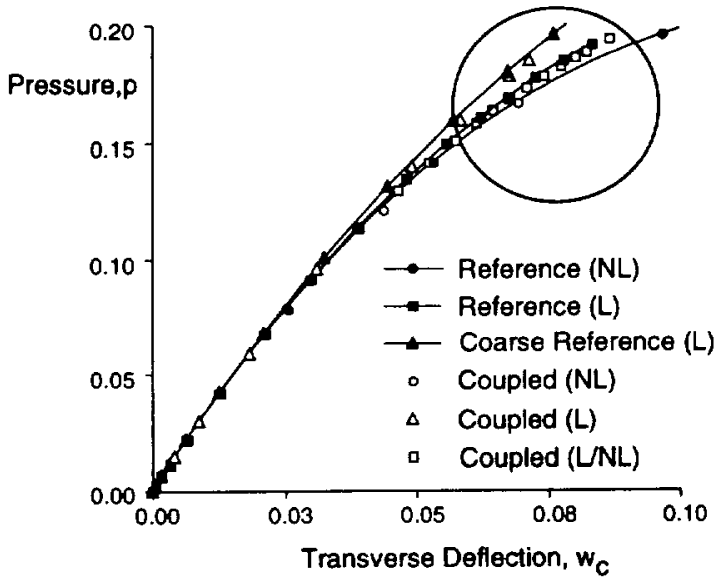

(a) Global View

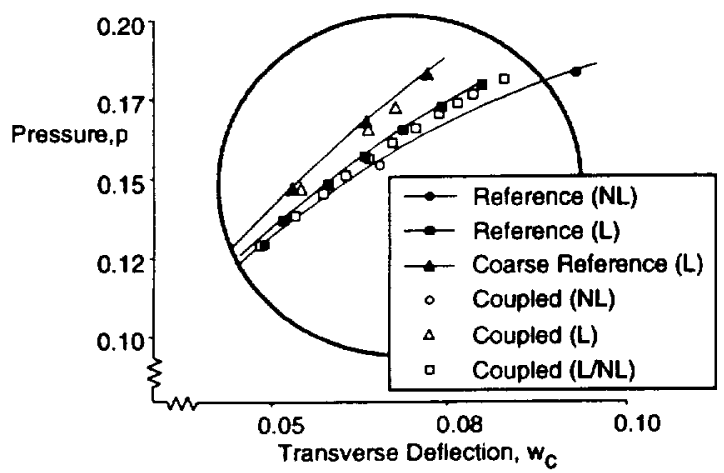

(b) Enlarged View of Encircled Region

Figure 9. Effect of Nonlinear Strain Approximations on Transverse Deflection.

Based on these results, nonlinear strain approximations are used in the coarse substructure while linear strain approximations are used in the refined substructure. The results of this analysis with combined linear and nonlinear strain approximations are given by the open squares in Figure 9 and are denoted by coupled (L/NL) in the figure. These results are bounded by the solutions using the refined reference model with linear strains and nonlinear strains (solid squares and circles in the figure, respectively). Computations with nonlinear strains are more expensive than those with linear strains due to the increased number of operations required in evaluating the nonlinear terms in the strain-displacement relations. Hence, a trade-off can be made between the level of mesh density and the level of nonlinear approximation. For this benchmark application, the results obtained with linear strains in the most refined substructure and nonlinear strains in the coarse substructure were more accurate than the results obtained with the refined reference model using linear strains only. These results demonstrate the unique analysis capability provided by the interface technology. Although in this example, the corotational formulation provided the nonlinear response character in each substructure, this concept may be extended to the application of linear/nonlinear substructuring in which one or more substructures are identified as exhibiting nonlinear behavior while the other substructures are assumed to exhibit linear behavior. In this case, the tangent stiffness matrices are computed for the nonlinear substructures and assembled along with the linear stiffness matrices for the linear substructures.

\section{Hinged Cylindrical Panel}

An isotropic cylindrical panel which is hinged on its two straight edges and free on its two curved edges (shown in Figure 10) and which is subjected to a concentrated load at its center is used to demonstrate the capability of the interface technology on a structure exhibiting collapse. This panel configuration is given in the literature and attributed to Sabir and Lock. ${ }^{21}$ The panel material properties are $3.10275 \mathrm{kN} / \mathrm{mm}^{2}(450 \mathrm{ksi})$ for the Young's modulus and 0.3 for the Poisson's ratio. The panel radius, $R$, is $2540 \mathrm{~mm}$. (100 in.); the panel half-length, L, is $254 \mathrm{~mm}$. (10 in.); and the half-opening angle, $\varphi$, is 0.1 radians. Two panel thicknesses, t, were considered: a thickness of $12.7 \mathrm{~mm}$. $(0.5 \mathrm{in}$.) and a thickness of $6.35 \mathrm{~mm}$. (0.25 in.), yielding radius-tothickness ratios, $R / t$, of 400 and 200 , respectively. Both the thick and thin panels exhibit a limit point and snap-through behavior (See Figure 11) as the load is increased, and the panels collapse into an inverted configuration. In addition, the thin panel exhibits a snap-back behavior (See Figure 12). The coupled finite element model used for the analysis of both panels is the same as that used for the clamped cylindrical panel and is shown in Figure 7 . The interface is located along an axial line at the panel halfopening angle, $\varphi$.

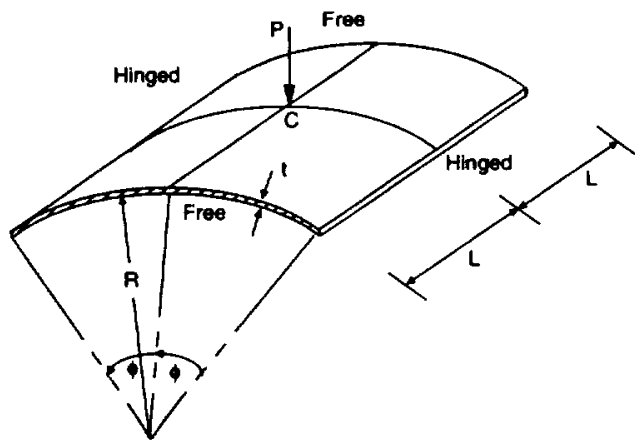

Figure 10. Hinged Cylindrical Panel Subjected to Concentrated Load. 
The concentrated load is given as a function of the transverse deflection at the center of the thick and thin panels (point $C$ in Figure 10) in Figure 11 and Figure 12, respectively. The response curve for the thick panel (see Figure 11) indicates the limit point and snap-through of the panel as the load is increased. The response curve for the thin panel (see Figure 12) indicates the snap-back behavior of the panel. The results with the interface element for both the thin and thick panels are in excellent agreement with the reference solution.

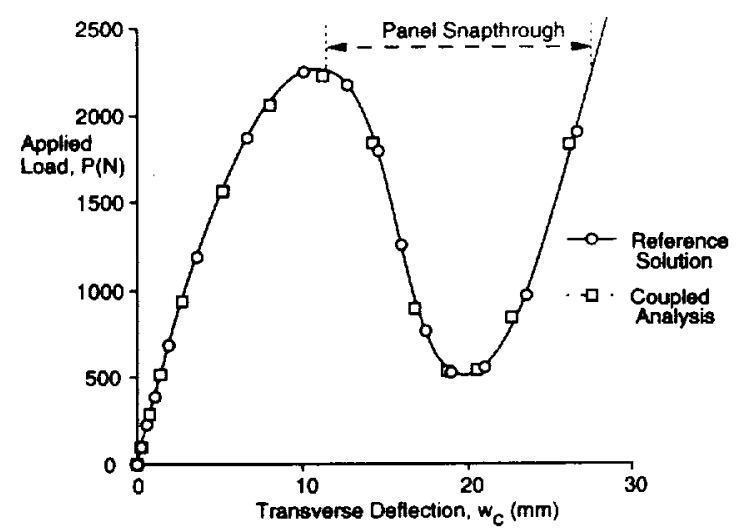

Figure 11. Central Deflection of Thick Hinged Cylindrical Panel.

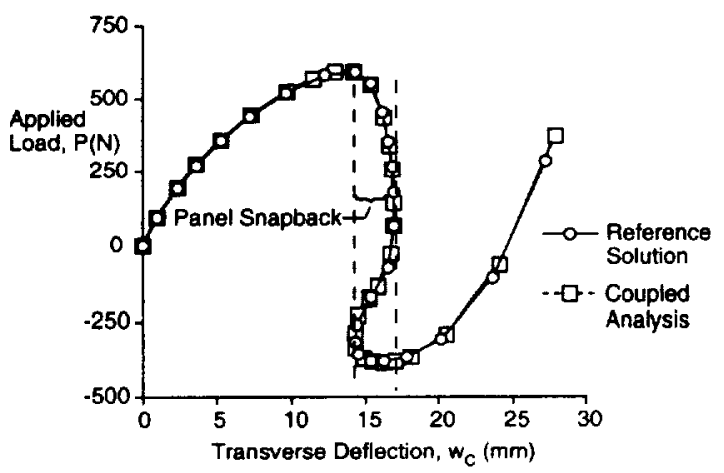

Figure 12. Central Deflection of Thin Hinged Cylindrical Panel.

\section{Cantilevered Plate in Pure Bending}

An isotropic rectangular plate (shown in Figure 13) subjected to an end bending moment is used to demonstrate the capability of the interface technology for a structure exhibiting very large rotations. The plate is clamped at its root, and, to emulate beam behavior, symmetry conditions are used on the long edges. This configuration is often referred to as the elastica problem. The plate material properties are 120 psi for the Young's modulus and 0.0 for the Poisson's ratio. The plate length, $L$, is 10 in., and the width, W, and thickness, $t$, are both 1 in. The coupled finite element model is shown in Figure 14 with the interface located at the panel midwidth. In Figure 15, the normalized moment is given as a function of the deflection at the loaded end of the plate normalized by the plate length. The results indicate the very large rotations which the structure undergoes, and the interface element analysis is in excellent agreement with the reference solution. The interface element performed well even for such large rotations.

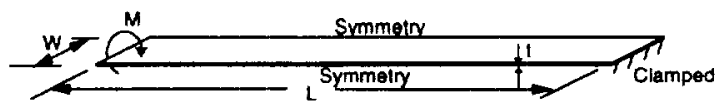

Figure 13. Cantilevered Plate Subjected to Pure Bending.

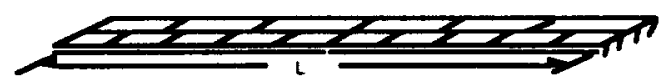

Figure 14. Finite Element Models for Cantilevered Plate.

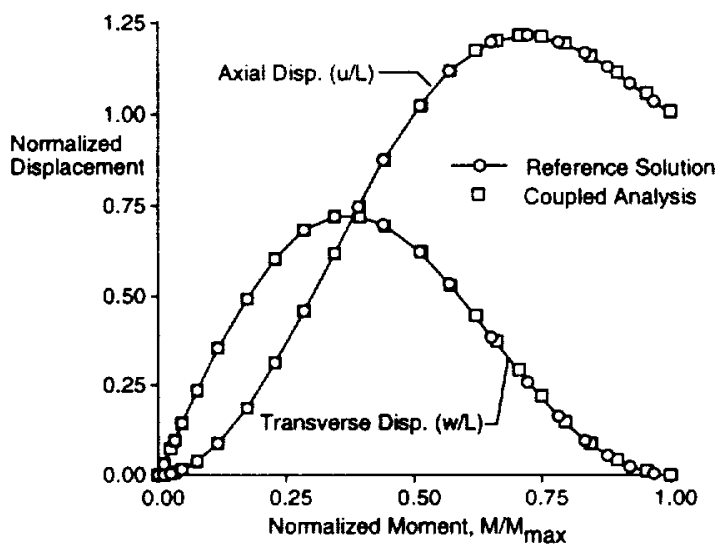

Figure 15. Deflection of Cantilevered Plate.

\section{Composite Panel}

A composite rectangular panel (shown in Figure 16) subjected to axial compression is used to demonstrate the capability of the interface technology in the postbuckling regime. This panel is clamped at the root and simply-supported by knife-edge supports on its long edges to prevent it from buckling as a wide column. The panel used is a 24-ply orthotropic laminate and is denoted Panel $\mathrm{C} 4$ in the experimental and analytical results reported by Starnes and Rouse. ${ }^{22}$ The panel is fabricated from unidirectional graphite-fiber tapes preimpregnated with $450 \mathrm{~K}$ cure thermosetting epoxy resin. The ply properties are $19,000 \mathrm{ksi}$ for the longitudinal Young's modulus, $1890 \mathrm{ksi}$ for the transverse Young's modulus, $930 \mathrm{ksi}$ for the in-plane shear modulus, $250 \mathrm{ksi}$ for the transverse shear modulus, and 0.38 for both Poisson's ra- 
tios. The laminate stacking sequence for the panel is $\left[ \pm 45 / 0_{2} / \pm 45 / 0_{2} / \pm 45 / 0 / 90\right]_{s}$, and the nominal ply thickness is 0.00551 in. The panel length, $\mathrm{L}$, is 20 in., and the width, $W$, is 6.75 in. The overall panel thickness, $t$, is 0.13 in. The reference model has 12 elements along the length and 6 elements along the width. The panel was observed in the test ${ }^{22}$ to buckle into two longitudinal half-waves and one transverse half-wave which resulted in peak stresses along its center. Thus, the region of interest for this application is in the vicinity of the center of the panel, and a refined model is used in that region.

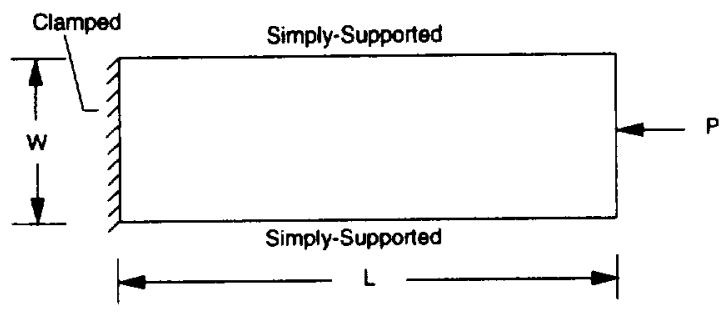

Figure 16. Composite Panel Subjected to Compressive Loading.

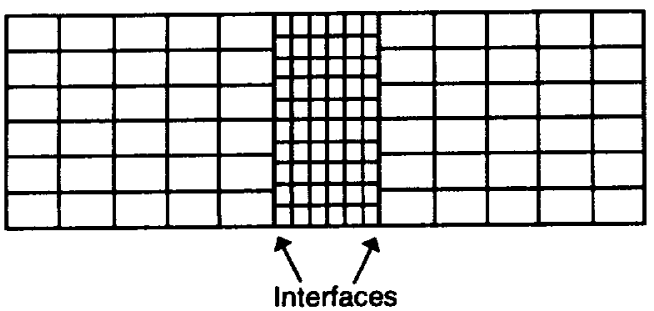

Figure 17. Finite Element Models for Composite Panel.

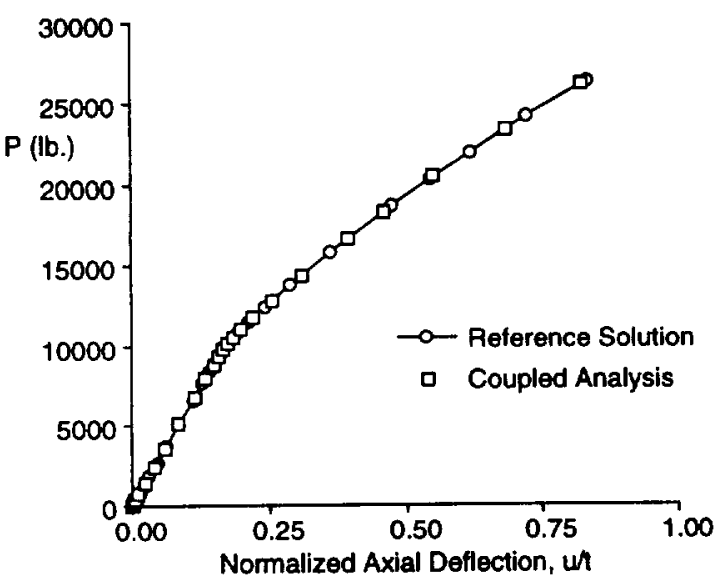

Figure 18. Axial End Deflection of Composite Panel.
The coupled finite element model is shown in Figure 17. Two interfaces are used in this analysis and are depicted by the gray shaded lines in Figure 17. The compressive load is given as a function of the axial deflection at the loaded edge and maximum transverse deflection normalized by the panel thickness in Figure 18 and Figure 19, respectively. The response curves indicate the softening of the panel as the load is increased. The postbuckling response exhibits large out-of-plane deflections (over three times the thickness, see Figure 19). The interface element analysis is in excellent agreement with the reference solution.

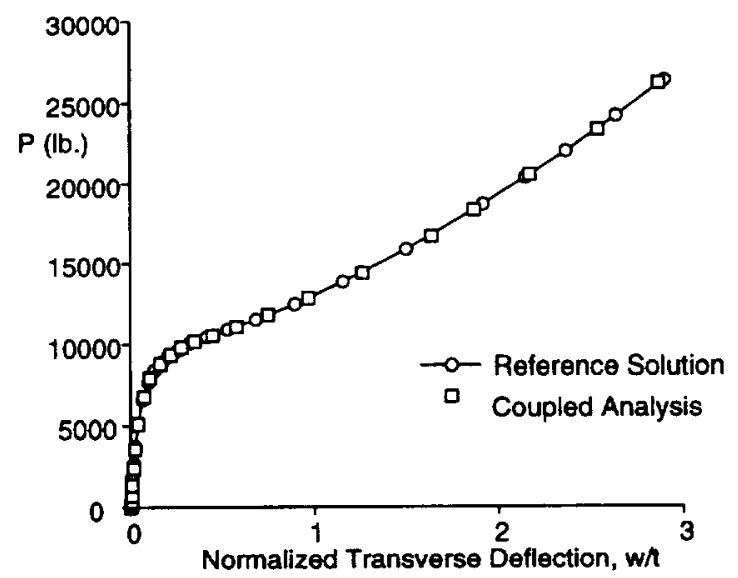

Figure 19. Maximum Transverse Deflection for Composite Panel.

\section{Concluding Remarks}

Interface technology for geometrically nonlinear analysis of multiple connected subdomains has been demonstrated. As in the case of the linear formulation, the subdomains need not be nodally compatible, and, thus, the need for potentially complex transition modeling is eliminated. A hybrid variational formulation was utilized to achieve compatibility, in a variational sense, between the independently modeled substructures. The development of the hybrid formulation in the form of an element facilitates the implementation of this approach in standard finite element software packages. The interface element stiffness is computed and assembled along with standard finite elements allowing for the use of standard finite element matrix assemblers and linear and nonlinear solution procedures.

The interface technology described herein has been demonstrated on several applications which exhibit a variety of response characteristics, namely: stiffening, softening, snap-through, snap-back, large rotations, and postbuckling. The applications used to demonstrate the capabilities of the interface technol- 
ogy for predicting such responses were a clamped square plate loaded with a uniform pressure, a clamped cylindrical panel loaded with uniform pressure, a hinged cylindrical panel with a concentrated transverse load, a cantilevered plate subjected to an end bending moment, and a composite panel loaded in axial compression. The results obtained using the interface technology were in excellent agreement with the reference solutions for all cases.

The capability of using different orders of strain approximations in the independent substructures has been demonstrated with the analysis of the clamped cylindrical panel. This unique analysis capability allows the analyst to specify the order of the straindisplacement approximation to be used in each substructure. Nonlinear strain approximations are computationally more expensive than linear strain approximations due to the increased number of operations required in evaluating the nonlinear terms in the strain-displacement relation. Hence, a trade-off can be made between the level of mesh density and the level of nonlinear approximation. In the case studied, the results obtained with linear strains in the most refined substructure and nonlinear strains in the coarse substructure were more accurate than the results obtained with the reference model approximated with linear strains only. In addition, as often done in nonlinear substructuring, the analyst may use the interface technology to isolate a region requiring nonlinear strain approximations while using the linear strain approximations elsewhere.

The interface technology described herein provides a method for predicting the nonlinear response of plate and shell problems as well as concomitant detailed stress states. The element has been implemented within a general-purpose finite element code.

\section{References}

1. Aminpour, M. A., Ransom, J. B., and McCleary, S. L., "Coupled Analysis of Independently Modeled Finite Element Subdomains," AIAA Paper Number 92-2235, 1992.

2. Ransom, J. B., McCleary, S. L., and Aminpour, M. A., "A New Interface Element for Connecting Independently Modeled Substructures," ALAA Paper Number 93-1503, 1993.

3. Whitcomb, J. D., and Kyeongsik, W., "Application of Iterative Global/Local Finite Element Analysis. Part 1: Linear Analysis," Communications in Numerical Methods in Engineering, Vol. 9, 1993, pp. 745-756.
4. Whitcomb, J. D., and Kyeongsik, W., "Application of Iterative Global/Local Finite Element Analysis. Part 2: Geometrically Nonlinear Analysis," Communications in Numerical Methods in Engineering, Vol. 9, 1993, pp. 757766.

5. Dodds, R. H., Jr., and Lopez, L. A., "Substructuring in Linear and Nonlinear Analysis." International Joumal for Numerical Methods in Engineering, Vol. 15, 1980, pp. 583-597.

6. Clough, R. W., and Wilson, E. L., "Dynamic Analysis of Large Structural Systems with Local Nonlinearities," Computer Methods in Applied Mechanics and Engineering, Vol. 17/18, Part 1, January 1979, pp. 107-129.

7. Storaasli, O. O., and Bergan, P., "Nonlinear Substructuring Method for Concurrent Processing Computers," AIAA Journal, Vol. 25, No. 6, June 1987, pp. 871-876.

8. Ransom, J. B., and Knight, N. F., Jr., "Global/Local Stress Analysis of Composite Panels," Computers and Structures, Vol. 37, No. 4, 1990, pp. 375-395.

9. Aminpour, M. A., McCleary, S. L., Ransom, J. B., and Housner, J. M., "A Global/Local Analysis Method for Treating Details in Structural Design," ASME AMD-Vol. 157, Adaptive, Multilevel, and Hierarchical Computational Strategies, edited by A. K. Noor, 1992, pp. 119-137.

10. Aminpour, M. A., Krishnamurthy, T. McCleary, S. L., and Baddourah, M. A., "Application of a New Interface Element to the Global/Local Analysis of a Boeing Composite Crown Panel," Fourth NASA/DoD Advanced Composites Technology Conference, June 7-11, Salt Lake City, UT, NASA CP-3229, compiled by J. G. Davis, Jr., J. E. Gardner, and M. B. Dow, Volume I, Part 2, 1993, pp. 773-788.

11. "MSC and NASA Agreement to Include NASA Technology in MSCINASTRAN," MSCWORLD, Volume V, No. 1, February 1995, pp. 23-24.

12. Schiermeier, J. E., Housner, J. M., Ransom, J. B., Aminpour, M. A., and Stroud, W. J., "The Application of Interface Elements to Dissimilar Meshes in Global/Local Analysis," Proceedings of the 1996 MSC World Users' Conference, Newport Beach, CA, June 3-7, 1992. 
13. Zienkiewicz, O. C., The Finite Element Method. Third Edition, McGraw-Hill Book Company, UK, 1977, pp. 304-328.

14. Stanley, G. M., Hurlbut, B., Levit, I., Stehlin, B., Loden, W., and Swenson, L., COMET-AR Adaptive Refinement Manual, LMSC Report \#F318482, 1991.

15. Riks, E., "On the Numerical Solution of Snapping Problems in the Theory of Stability," August 1970, Stanford University, SUDAAR Report No. 401. Also available as AFOSR Report No. 70-2258TR.

16. Wempner, G. A., "Discrete Approximations Related to Nonlinear Theories of Solids," International Journal of Solids and Structures, Vol. 7, 1971, pp. 1581-1589.

17. Rankin, C. C., and Brogan, F. A.: "An ElementIndependent Corotational Procedure for the Treatment of Large Rotations." In Collapse Analysis of Structures, edited by , L. H. Sobel and K. Thomas, ASME, New York, 1984, pp. 85100.

18. Crisfield, M. A., Nonlinear Finite Element Analysis of Solids and Structures - Volume I: Essentials, John Wiley \& Sons, NY, 1992.

19. Park, K. C., Stanley, G. M., “A Curved C Shell Element Based on Assumed Natural-Coordinate Strains," ASME Journal of Applied Mechanics, Vol. 108, 1986, pp. 278-290.

20. Brebbia, C. A., and Conner, J., "Geometrically Nonlinear Analysis," Proc. Am. Soc. Civ. Eng., Vol. 95, EM2, 1969, pp. 463-468.

21. Sabir, A. B., and Lock, A. C., "The Application of Finite Elements to the Large Deflection Geometrically Nonlinear Behavior of Cylindrical Shells," Proceedings of the International Conference on Variational Methods in Engineering, University of Southampton, Southampton, England, Session 7, September 1972, pp. 7/67-7/76.

22. Starnes, J. H., Jr., and Rouse, M., "Postbuckling and Failure Characteristics of Selected Flat Rectangular Graphite-Epoxy Plates Loaded in Compression," AIAA Paper No. 81-0543, 1981. 
